Cahiers $d u$ MONDE RUSSE

\section{Cahiers du monde russe}

Russie - Empire russe - Union soviétique et États indépendants

$51 / 4 \mid 2010$

Sciences humaines et sociales en Russie à l'Âge d'argent

\title{
Wendy Z. Goldman, Terror and Democracy in the Age of Stalin
}

\section{François-Xavier Nérard}

\section{OpenEdition}

\section{Journals}

Édition électronique

URL : https://journals.openedition.org/monderusse/7389

DOI : $10.4000 /$ monderusse.7389

ISSN : $1777-5388$

Éditeur

Éditions de l'EHESS

\section{Édition imprimée}

Date de publication : 25 novembre 2010

Pagination : 713-716

ISBN : 978-2-7132-2316-7

ISSN : $1252-6576$

Référence électronique

François-Xavier Nérard, « Wendy Z. Goldman, Terror and Democracy in the Age of Stalin », Cahiers du monde russe [En ligne], 51/4 | 2010, mis en ligne le 09 décembre 2011, consulté le 03 septembre 2022. URL : http://journals.openedition.org/monderusse/7389; DOI : https://doi.org/10.4000/ monderusse.7389

Ce document a été généré automatiquement le 3 septembre 2022.

Tous droits réservés 


\title{
Wendy Z. Goldman, Terror and Democracy in the Age of Stalin
}

\author{
François-Xavier Nérard
}

\section{RÉFÉRENCE}

Wendy Z. GoldMAN, Terror and Democracy in the Age of Stalin. The Social Dynamics of Repression. Cambridge - New York : Cambridge University Press, 2007, 274 p.

1 Wendy Goldman appelle de ses vœux - c'est d'ailleurs le titre de son introduction - une « histoire sociale de la terreur ». L'ambition n'est pas totalement neuve: elle se situe clairement dans le prolongement de la réflexion des auteurs dits "révisionnistes » auxquels elle se réfere. Les nouvelles sources disponibles de l'histoire de la répression stalinienne (notamment sur les grandes opérations de masse) ont pour un temps concentré l'attention sur les pratiques policières de la terreur et sur son organisation par l'appareil répressif d'État. L'aspect plus «social» - notamment la question de la participation de la population - a été nettement plus négligé.

2 Le livre conduit cependant à s'interroger sur la définition de la « terreur ». Les historiens utilisent, depuis Robert Conquest en 1968' , ce mot passe-partout sans jamais chercher réellement à le définir avec précision. Pourtant, depuis l'invention de la notion, celle-ci s'est profondément complexifiée. Veut-on parler de la vague de répression contre les élites (présentes et passées) du régime, qui débute avec le meurtre de Kirov en 1934 ? Pense-t-on à l'élimination le plus souvent physique des cadres intermédiaires (responsables régionaux et locaux) au cours de 1936-1938 ? Évoque-t-on les grands procès de Moscou et la multitude de procès du même type en province? Ou songe-t-on plutôt aux opérations de masse organisées par les décrets opérationnels de Ežov à l'été 1937 ? La terreur stalinienne est-elle tout ou partie de ces composantes?

3 La question est évidemment complexe, mais elle ne cesse de préoccuper le lecteur tout au long de l'ouvrage. Wendy Goldman néglige largement les opérations de masse sur lesquelles l'historiographie se concentre pourtant depuis plusieurs années. Elle ne les 
mentionne qu'en fin d'ouvrage (p. 209) et choisit en revanche de se focaliser sur la face visible de la terreur, notamment celle qui concerne les responsables des syndicats et du parti au niveau local. L'idée centrale est dès lors de cerner la participation populaire aux mécanismes répressifs. Pour l'auteur, ce n'est pas seulement le nombre des victimes qui fait la terreur, « c'est également le nombre de perpetrators qu'elle engendre » (p. 7). Le texte pose donc le problème de la participation et de la complicité de la population. Wendy Goldman semble opter pour une analyse en termes de consentement et de soutien, forgés à moyen terme par une politique volontariste du pouvoir stalinien. Il faut pourtant s'interroger sur le poids de cette participation et sur sa nécessité dans le processus répressif. Autrement dit, sans cette participation, pourrait-on parler de terreur? La terreur stalinienne s'appuie-t-elle sur ces pratiques à la base, en a-t-elle besoin pour se développer? Ces questions sont finalement assez peu abordées dans l'ouvrage, tant l'auteur semble considérer que le lien entre participation et répression est établi.

5 Le livre est organisé autour de six chapitres qui font alterner une étude des mécanismes du pouvoir au sommet et à la base. Il s'ouvre par une partie introductive, assez classique, qui montre un monde du travail mis sous pression par la politique stalinienne, où le fossé entre la classe ouvrière et le pouvoir qui est censé la représenter ne cesse de s'élargir. Les chapitres III, IV et V étudient la structuration de la politique répressive au centre et proposent une vision de la terreur qui reste somme toute assez traditionnelle, en traçant un continuum entre le meurtre de Kirov et les procès de Moscou (p. 55-56). Ils se fondent pour l'essentiel sur des données recueillies dans les archives centrales, notamment celles du Comité central des syndicats (VTsSPS). Les deux chapitres qui les encadrent (II et VI) relèvent en revanche d'une analyse des mécanismes de terreur et de répression à la base et mobilisent des archives de plusieurs comités du parti des usines de Moscou.

6 Cette étude à la base est sans conteste la plus intéressante et la plus stimulante de l'ouvrage. Wendy Goldman souligne notamment l'attitude pour le moins sceptique des membres du parti face aux tentatives d'imposer une vision du monde en termes de sabotage, une approche des problèmes où le dysfonctionnement n'en est pas un mais le fruit de la main de l'ennemi (p. 74-75, par exemple). Elle montre bien comment la figure du « zinoviévo-trotskiste » peine à s'imposer. C'est un point qui semble important. On avait pu constater une résilience du même type lors du lancement de la campagne sur l'autocritique en 1928. Les discours officiels étaient plus accueillis par des sarcasmes que par un soutien aux nouveaux mots d'ordre.

7 Il faut du temps au pouvoir stalinien pour obtenir un semblant de réaction, une participation active aux répressions, et Wendy Goldman décrit les étapes de cette soumission. Elle insiste notamment sur plusieurs moments clés, notamment le deuxième grand procès de Moscou où les travailleurs sont désormais présentés comme la cible des ennemis du pouvoir soviétique (p. 106). Pour autant, axée sur 1936-1937, l'auteur néglige le fait que la figure du traitre et du saboteur qu'elle décrit (« la paille dans le pain, les chevaux morts, l'échec à tenir les objectifs de production, tout cela avait un nouveau nom : c'était appelé le sabotage», p. 51) n'est pas une nouveauté. Le principe de la responsabilité individuelle du dysfonctionnement - et donc de son acceptation ou de son refus par les Soviétiques - a une histoire qui remonte au moins à 1928. L'étude que Goldman propose, dans le troisième chapitre (p. 96-104), du procès consécutif au désastre de Kemerovo est très intéressante, mais on peut aussi y voir une sorte de rejeu du procès 
de Šahty... Ce n'est donc probablement pas un hasard si le mot d'ordre de l'autocritique, tombé dans un oubli relatif depuis 1929, connait un rebond important en 1937.

Une hypothèse sous-tend les analyses de Wendy Goldman - celle d'un système où le pragmatisme, voire le cynisme, sont la norme : du côté du pouvoir, ainsi qu'en témoigne l'utilisation par les staliniens du meurtre de Kirov, mais aussi justement des accidents de Kemerovo, tout comme au sein de la population. Les Soviétiques semblent savoir «parler bolchevik» (voir stalinien) quand cela les arrange. Curieusement, Goldman mène cette analyse jusqu'en 1937, considérant que, cette année-là, les efforts staliniens portent leurs fruits et que, dans un mélange de peur et d'intérêt, les membres des syndicats et du parti finissent par croire à ce dont ils doutaient la veille encore. Le basculement de 1937 vers la complicité répressive aurait pourtant pu être interprété en termes d'effets d'aubaine de la part des simples Soviétiques qui auraient su jouer le rôle qu'on attendait d'eux quand leurs intérêts entraient en jeu, quand les possibilités de revanche sociale, ou hiérarchique, apparaissaient...

9 Au cœur de cette étude, on trouve les élections en vue du renouvellement des dirigeants syndicaux de l'été 1937. L'auteur les présente comme un moment-clé. Ce sont elles qui, dans une large mesure, expliquent le titre surprenant et provocateur de l'ouvrage qui rapproche terreur et démocratie. Ces élections ont lieu à bulletin secret et plusieurs candidats sont en lice, même si Goldman néglige de préciser que chaque candidat doit être autorisé à se présenter par un vote à main levée, ce qui limite quand même le potentiel « démocratique » de ces élections. Le plénum de février-mars 1937 n'est ainsi pas étudié comme le dernier de Buharin, mais - de façon plus originale - comme celui du lancement de ces élections syndicales. Le lien entre élections et répression est clairement affirmé : « Moins d'un mois plus tard, les principes de la démocratie promulgués par le plénum allaient mettre les syndicats sens dessus dessous et provoquer une course frénétique pour le pouvoir et la survie » (p. 112).

10 Il semble toutefois que le trait soit un peu forcé et que ces élections se soient déroulées bien plus paisiblement que Goldman ne semble le suggérer. L'hypothèse que l'historienne propose dans le quatrième chapitre est néanmoins intéressante, sans parvenir à être totalement convaincante. Goldman montre la complexité de ces élections qui ne font pas accéder aux responsabilités des hommes nouveaux mais relèvent plus certainement d'un jeu de chaises musicales. Ce chapitre reste malheureusement fondé sur des sources centrales, forcément globalisantes. On aurait aimé que l'auteur aille voir sur le terrain ce qu'il en est des processus électoraux qu'elle décrit. Pour avoir consulté certains protocoles de façon non systématique, l'auteur de ces lignes en a retiré une impression légèrement différente, celle d'une atmosphère beaucoup moins tendue, hétérogène en tout cas. Alors que le lecteur s'attend à une plongée au plus près des acteurs, le chapitre $\mathrm{V}$ retourne à une analyse plus classique des événements au centre, analyse la répression des dirigeants syndicaux avec une chronologie d'ailleurs légèrement différente puisqu'elle oblige l'auteur à revenir à 1936... La question est pourtant d'importance. La thèse de Goldman est en effet que le poids numérique important des syndicats - en bref le nombre de Soviétiques concernés - en aurait fait un vecteur efficace de la terreur. L'idée est séduisante, mais mériterait une démonstration plus poussée.

11 Le dernier chapitre est bien consacré aux répressions dans les usines, mais il se fonde sur les archives des comités du parti de cinq usines moscovites (1937-1939). C'est donc le parti qui est étudié, plus que les syndicats. Or, les populations concernées ne sont pas les 
mêmes, ni en volume ni en qualité. Cette dernière section, très intéressante, interroge également la participation de la population à la répression, notamment par l'intermédiaire des dénonciations, orales ou écrites. Goldman y propose une étude thématique intéressante et complète (trous noirs des biographies, origine sociale, cercles de relations) des attaques portées. Mais il nous semble que, là encore, la question de la nécessité de ces dénonciations dans le processus répressif devrait être posée. La réalité des dénonciations est un fait difficilement contestable : chaque mise en accusation d'un responsable ou d'un simple membre du parti, chaque "fragilisation" provoquait de nombreux «signaux». Ceux-ci étaient-ils pour autant indispensables au processus répressif?

12 Wendy Goldman offre avec ce livre une étude intéressante des mécanismes de la terreur stalinienne, qui suscite de nombreuses réflexions sans pour autant emporter totalement la conviction. Le lien établi dans le titre entre « démocratie » et « terreur» ne semble pour le moins pas évident à la lecture de l'ouvrage.

\section{NOTES}

1. Robert Conquest, The Great Terror: Stalin's Purge of the Thirties, New York: Macmillan, 1968, $633 \mathrm{p}$. 